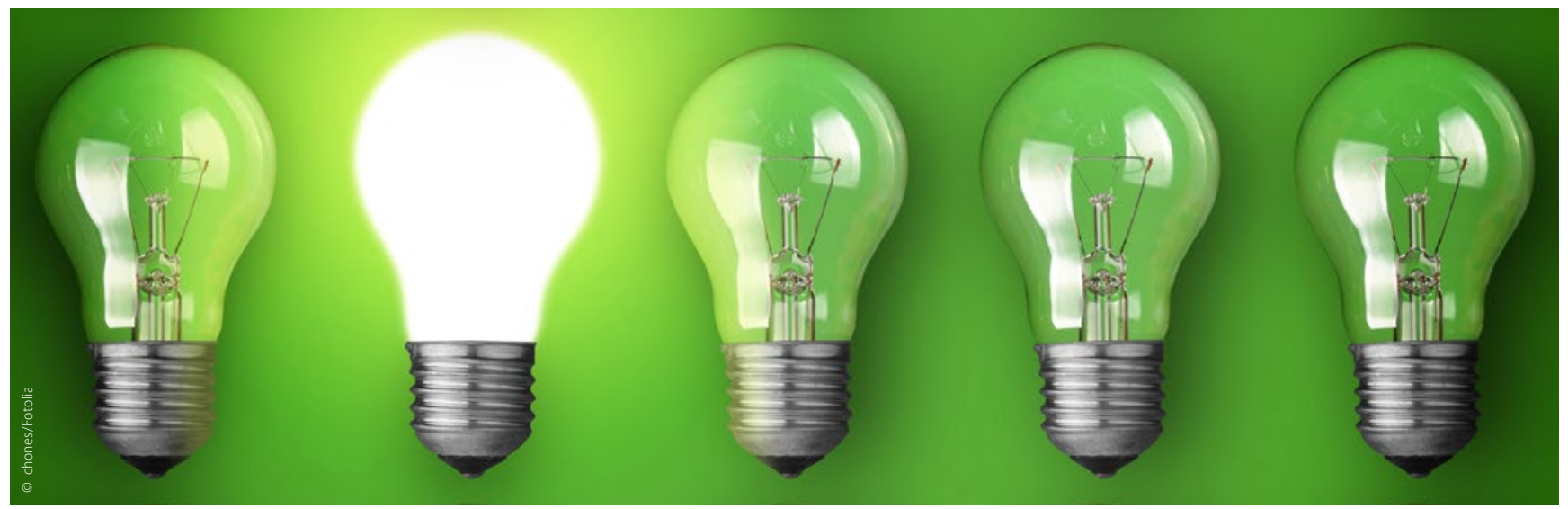

\title{
Photodynamische Therapie
}

\section{Erhellende Behandlung der Periimplantitis?}

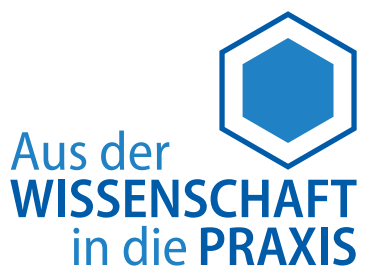

Eine bakterielle Besiedlung von Oberflächen umgibt uns allgegenwärtig. Insbesondere in der bakteriell kontaminierten Mundhöhle kann eine bakterielle Besiedlung, die mit der Bildung eines sogenannten Biofilms einhergeht, zu Entzündungen führen. Insbesondere an den oft angerauten Titanoberflächen dentaler Implantate bildet sich ein derartiger Biofilm.

Die daraus entstehende Periimplantitis kann zu einem meist irreversiblen Knochenabbau führen. Die Entzündung der periimplantären Mukosa ist bei der Behandlung hingegen reversibel. Zur Behandlung der Periimplantitis können chirurgische von nichtchirurgischen Techniken unterschieden werden. Bei den nichtchirurgischen Techniken werden die klassischen mechanischen Methoden von nichtmechanischen unterschieden. Eine Neuerung auf diesem Gebiet ist in einer der vergangenen Ausgaben dieser Zeitschrift bereits beschrieben worden: die sogenannte „GalvoSurg“-Behandlung, bei der ein Galvanostrom an das Implantat angeschlossen wird, um den Biofilm aufzulösen. Diese Therapie ist allerdings aktuell noch nicht kommerziell erhältlich.

Anders verhält es sich mit der seit Langem angewandten Pulverstrahlreinigung (AirPolishing) oder der photodynamischen Therapie, zum Beispiel mit Er:Yag-Lasern. In klinischenStudien (Renvert et al. 2010) konnten beide Methoden das Bleeding on Probing (BOP) reduzieren. Im Ergebnis unterschieden sich die beiden Gruppen allerdings nicht. Verglichen mit der konventionellen, abrasiven Reinigungsmethode scheint die Pulverstrahlreinigung nicht überlegen zu sein. Insbesondere in Kombination mit lokalen Antibiotika konnte mit der konventionellen mechanischen Reinigung der Implantatoberflächen das BOP reduziert werden.
Ein weiterer Ansatz der photodynamischen Therapie: Sie nutzt photosensibilisierende Substanzen wie Toluidinblau oder Chlorin e6 (Ce6), die per Diodenlaser aktiviert werden. In Tierversuchen ist 2005 schon durch die Kombination von Toluidinblau und einem GaAlAs-Diodenlaser $(830 \mathrm{~nm})$ von einer Knochenaugmentation an freiliegenden Implantatwindungen berichtet worden (Shibli et al. 2005). In diesem Jahr ist eine Methode publiziert worden, die mit sichtbarem und wassergefiltertem Infrarot-A-Licht (Visible Light Plus Water-Filtered Infrared A, VIS+wIRA) in Kombination mit Toluidinblau beziehungsweise Ce6 die Bakterienlast an im Mund befindlichen Kunststoffmaterialien reduzieren konnte.

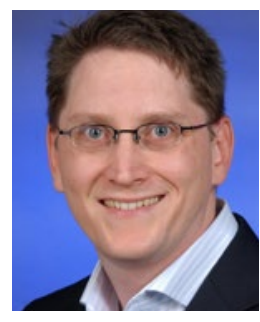

Felix P. Koch

Autor des Wissenschaftlichen Info-Dienstes, stellvertretender Klinikdirektor und Leitender Oberarzt für Mund-, Kiefer- und Gesichtschirurgie der Uniklinik Frankfurt/Main. 